\section{LA MALARIA IN UMBRIA TRA STORIA E LEGGENDA DANIELE CROTTI}

\section{Il parte}

\section{La malaria dell'area del lago Trasimeno}

La realtà territoriale del Lago Trasimeno fornisce un esempio piuttosto eclatante della atavica lotta ambiente-uomo, nella misura in cui per lunghi secoli le popolazioni del lago hanno dovuto fare $\mathrm{i}$ conti con un lago nemico-amico che troppe volte, invadendo con le sue acque $\mathrm{i}$ territori adiacenti, finiva col trasformarli in estesi pantani e causava tra la gente febbri e morte (3, 38).

Di origine, come detto, antichissima, e chiamata in causa tra i fattori che avrebbero decretato la fine di importanti civiltà del passato (forse gli stessi Etruschi, ad esempio), la malaria, anche nell'area del Trasimeno, compare in epoca remota. A dispetto di quegli studiosi e scrittori che nel corso dei secoli hanno negato la presenza di aria insalubre e "ammorbata", la maggior parte delle testimonianze storiche hanno riportato attendibili notizie sulla presenza di febbri periodiche e "perniciose" nell'area del Trasimeno $(30,38)$.

La malaria fu presente fin dalla più remota antichità nelle zone tropicali, subtropicali ed in quelle temperate del globo terrestre. L'area mediterranea è stata da sempre tra le regioni maggiormente colpite e l'Italia in modo particolare. Nell'Italia Centrale un triste primato è spettato alla Maremma Toscana, alle Paludi Pontine, ed anche ai territori soggetti agli straripamenti del fiume Tevere, nonché, e non per ultimo, all'area trasimenica, sia pur in misura assai più contenuta sia nello spazio che nel tempo (38).

Ambiente, salute e qualità della vita dunque:"Nel 1834 il Governatore di Castiglion del Lago ottenne licenza dal Delegato Apostolico di Perugia di trasferire il suo tribunale durante il periodo più caldo a Panicale e di poterci risiedere egli stesso, per ragione della malaria, che tremendamente e tristemente spopola le sponde del Trasimeno" (2). Ma prima ancora, e siamo nel I625, due secoli innanzi, Papa Urbano VIII aveva fatto trasferire i monaci, residenti nella Rocca dell'lsola Polvese (una delle due isole abitate del Lago), presso il monastero di S. Antonio a Porta Sole in Perugia, a causa, appunto, del clima umido e della malaria.

II Trasimeno è un lago chiuso, sprovvisto cioè di un emissario che per via naturale consenta di mantenere in equilibrio il livello delle sue acque. Per ovviare a tale deficienza sono state messe a punto, sin dall'epoca etrusca e poi romana, importanti opere di sistemazione idraulica, per fugare lo spettro delle esondazioni, da cui residui paludosi e febbri malariche (38).

Nel XV secolo anche Braccio Fortebraccio da Montone attuò un'opera idraulica in tale zona, ma senza grossi risultati positivi. Le piene e le inondazioni delle campagne perilacustri si susseguirono con ritmo a volte preoccupante, tanto che a partire dalla fine del
XVIII secolo furono avanzate varie proposte di intervento. Si delinearono sin da subito due gruppi contrapposti: uno rappresentato dai fautori del prosciugamento e l'altro costituito da quello dei sostenitori di un razionale risanamento da realizzarsi prima di tutto con la costruzione di un nuovo efficiente emissario (38). Nel 1779, ad esempio, Annibale Mariotti, "Pubblico Professore di Medicina Teorica e Protomedico Generale di Perugia", presentò una lunga relazione scritta circa il progetto del disseccamento del Lago, ponendosi fortemente contro. II Mariotti, con argomentazioni "fisico-mediche", mise in guardia dall'assurdità di tale ipotesi, pur non negando l'evidenza di infezioni febbrili periodiche legate all'aria e all'acqua insalubri. In tali "riflessioni" A. Mariotti mette però in ancor più evidenza come il mantenimento del Lago darebbe più vantaggi che svantaggi alla popolazione residente, qualora adeguatamente semplicemente "bonificato", senza quindi procedere al drastico prosciugamento (22). Di seguito alcuni estratti di queste sue "riflessioni". Se è vero che per palude si intende "uno spazio di terra ampio, ..., occupato da acque, ..., che sono per lo più torbide, e limacciose, che vengono ingombrate da vari insetti,... ed erbe palustri d'indole settica, e che ...tramandono fetore ...", è allora vero che se "si dia un'occhiata al Trasimeno" ... "si vede come le sue acque non possono rimproverarsi di questi difetti” (22). “... E lo spiacevole odore ... non basta a provare che sia seguita nell'acqua medesima una depravazione, e un guasto si grande da riempir l'aria di pestifere esalazioni". Ancora: "Ma se per tutte queste inondazioni si sa che restarono danneggiati le sementi, i campi, i villaggi; di niuna però, si dice, che cagionasse infezione d'aria e producesse epidemia, o mortalità degli abitanti”. E prosegue: "Gli abitatori di questa riviera sono senza dubbio soggetti a quelle malattie endemie che son più frequenti tra gli abitanti delle campagne, ... Non si pensi però che queste ... sia qui più universali, ... e più perniciose che altrove ... le febbri intermittenti, le putride, ..., sono assai volte meno frequenti, e men gravi intorno al Lago che nella Teverina, o in altri parti delTerritorio ...”. Indi:“Ma poiché la Paludi qual più e qual meno son però tutte nocive, e le acque loro, come le disse Ippocrate, (d) ad rem omnem improbae sunt, non è da porre in dubbio; .... Nel nostro Lago però niun male ... può consigliare a tentarne il disseccamento,..." (22).

$\mathrm{Nel}$ XIX secolo si riteneva che l'aria infetta fosse satura di minutissimi corpuscoli, nocivi alla salute umana, che si pensava prodotti dal calore e dall'umidità e sollevati da terra durante le ore diurne, mentre dopo il tramonto ripiombavano verso il basso "piovendo in quelle ore un malefico influsso" (30). In particolare ad essere temuti erano i mesi estivi, quando le acque, a causa del calore e dell'evaporazione, si ritiravano dai terreni inondati nei mesi precedenti. II culmine veniva raggiunto a settembre, quando si contava il maggior numero di casi da malaria (30).

Negli anni immediatamente successivi all'unità d'Italia, riprese vigore, però, il movimento d'opinione tendente ad imporre l'idea del prosciugamento del lago Trasimeno come la più idonea per combattere piene e straripamenti, debellare la malaria e favorire l'agricoltura (20).

Ma inquadriamo il problema malaria e Lago Trasimeno nel corso di questo XIX secolo, prima, durante e dopo 


\section{RASSEGNA}

la scoperta dell'eziologia della malattia parassitaria e del ruolo fondamentale svolto dalle zanzare. Se il bilancio delle esondazioni del lago e dei problemi idrici ad esso collegati nel sessantennio |800-|86| è degno di nota, altrettanto singolare si mostra il bilancio delle malattie e delle epidemie che colpirono le popolazioni laghigiane nella prima metà di tale secolo. Le tabelle sanitarie redatte dalla Deputazione Sanitaria ogni 15 giorni, a partire dal 1845 , riportavano saltuariamente notizie sulle febbri intermittenti, soprattutto quando queste colpivano con maggiore violenza. Per alcuni anni è possibile seguire passo a passo lo sviluppo della malaria, che seguiva un andamento "parabolico": iniziava a fare le sue prime vittime in primavera, per continuare fino all'inizio dell'autunno, toccando le punte massime ad agosto e settembre (30).

Va ricordato come per tutto questo secolo parlare di Trasimeno significava parlare di malaria, di febbri intermittenti e perniciose, di "esalazioni miasmatiche", che, secondo i medici e gli scienziati del tempo, ammorbavano l'aria che si respirava e rendevano invivibile il territorio adiacente al lago, soprattutto durante il secondo semestre dell'anno (38). Nelle fasi di più accentuata dilatazione delle acque del lago era probabile una successiva manifestazione di malaria di ampie proporzioni ed inevitabilmente i due fenomeni venivano messi in stretto rapporto di dipendenza. Così accadde nel '49, quando Castiglione del Lago fu colpita da "febbri periodiche e perniciose in molto numero, occasionate dal ritiro delle acque del lago, che putrefece gli animali e vegetabili...", ed ancora nel '55 quando, benché fosse ancora lontana la stagione estiva, l'ingrandimento delle paludi del Trasimeno stava favorendo lo sviluppo della malattia (30).

Per tutto il corso dell'ottocento aumentò lo stato di confusione tra le teorie mediche sull'eziologia della malaria, ma si iniziava, col passar degli anni (entriamo nella seconda metà del secolo), a respingere la spiegazione miasmatica e si faceva gradualmente strada la teoria parassitaria, anche per analogia con altre malattie, la cui comparsa era spiegata attraverso la presenza di insetti responsabili con la loro puntura dell'insorgere dell'epidemia. La scoperta poi del Plasmodium a fine secolo, anche se accolta inizialmente con riserve, determinò un balzo in avanti importante nel modo di concepire i rapporti epidemia-ambiente (38).

$\mathrm{Ma}$ quali sono gli aspetti peculiari del suddetto "ambiente malarico" e più in particolare perché anche l'area trasimenica in passato è stata terreno di ricorrenti epidemie contagiose di tal natura? Le aree geografiche a più alto rischio malarico sono quelle che più facilmente permettono alle zanzare anofele di crescere, vivere e riprodursi. L'ambiente malarigeno è quello che fornisce alle zanzare, nella fase larvale, gli elementi necessari per sopravvivere: umidità dell'atmosfera, quote altimetriche basse o molto basse, presenza di acque ristagnanti, ... . Tali fattori sono facilmente rintracciabili nell'area del Lago Trasimeno, per tutto il secolo diciannovesimo. Aggiungasi, tra le cause indirette, altri fattori quali le precarie condizioni igieniche delle aree abitate, la coltivazione di piante tessili (canapa e lino) la cui macerazione contribuiva a creare un ambiente propizio alle zanzare, la presenza di vaste zone occupate da canneti ed erbe palustri, tra i quali le anofele ben si sviluppano e si riproducono (Figura IV).
Se innegabile è così la presenza della malaria al Trasimeno, molto difficile è peraltro valutare in termini quantitativi il grado di incidenza del morbo sulla popolazione. Tra l'altro il termine "mal'aria" era poco utilizzato; si preferiva infatti ricorrere a dei sinonimi come "febbri intermittenti e perniciose" o "febbri periodiche e stagionali", che potevano riferirsi anche ad altro. All'inizio degli anni '60 del XIX secolo, secondo Innamorati (medico di Panicale), i centri più malsani dovevano essere quelli di Castiglione del Lago e di Borghetto, lungo la riviera occidentale, e di Montebuono, più verso Perugia (16). Tal F. Natali, in occasione di un'escursione intorno al lago, riguardo Borghetto ebbe modo di osservare: "quivi il Trasimeno incomincia a produrre cattivi effetti sull'atmosfera, imoperciocché stante la posizione del terreno che lo circonda, di sua natura basso e piano, produconsi al ritirarsi delle acque degli impaludamenti, non tanto micidiali però, giacché la superficie è costituita ovviamente di silice pura" (24). E di Castiglione: "Se nella stagione invernale e tiepida vi si respira un'aria discretamente sana, nell'estate questa è sì malsana che costringe gli abitatori ad emigrare. La malaria è prodotta dagli impaludamenti che si formano a destra e sinistra della penisola, giacché il lago ritirandosi lascia una plaga di terreno, che è come una palude, ove le canne palustri e le alghe vi si decompongono ammorbando di miasmi l'atmosfera" (24). Come si evince, ancora siamo nella fase dei "miasmi", per cui la malaria è conseguenza della putrefazione delle acque stagnanti. In ogni caso a metà secolo (e poco dopo) il bilancio dei malati di malaria doveva essere ogni anno piuttosto elevato: così, a Castiglione su 3.200 persone annualmente si contavano dai 1.800 ai 2.000 malati, di cui 300 colpiti in modo letale; nel comune di Tuoro, su 2.500 persone 850 erano vittime della malaria; in quello di Passignano si contavano 600 malati su 2.000 abitanti; in quello di Magione 260 su I.300 persone (38).

Passano gli anni e una enorme alluvione, verificatasi nel 1875, "una delle più vaste, durevoli e perniciose che si ricordino", arrecò notevoli danni e fece riprendere il dibattito tra le forze politiche e sociali sugli interventi da effettuare sul lago. I precedenti più grossi eventi calamitosi legati alla malaria a partire dalla metà del XVIII secolo alla fine del XIX, sono stati registrati nel I77I, nel I803, nel I830, nel I834, nel I849, nel I855, nel 1864 e, come appena riferito, nel biennio $1875-76$, a partire dall'autunno 1875 .

Sulla scia di tale dibattito, per iniziativa di Guido Pompili, che ottenne l'appoggio di molti proprietari terrieri circumlacuali, si iniziarono le pratiche per la creazione del "Consorzio per la sistemazione del Trasimeno" (28).

Lo stesso Pompili scrisse che nell'estate del 1876 il $10 \%$ delle persone era stato colpito dalla malaria e nelle cinque farmacie dei comuni attorno al lago erano stati venduti più di 15 chili di chinino, consumo di chinino che avveniva in dosi sostenute in quegli anni (38; Figura V). La cura sistematica delle febbri intermittenti contribuì certo alla graduale scomparsa della malaria, ma occorrerà attendere le trasformazioni socio-economiche del secolo prima di vederne la totale scomparsa e, nel caso specifico del Trasimeno, il risanamento ambientale con la costruzione del nuovo emissario (30).

Insomma, nel corso di un ventennio ricco di colpi di 


\section{RASSEGNA}

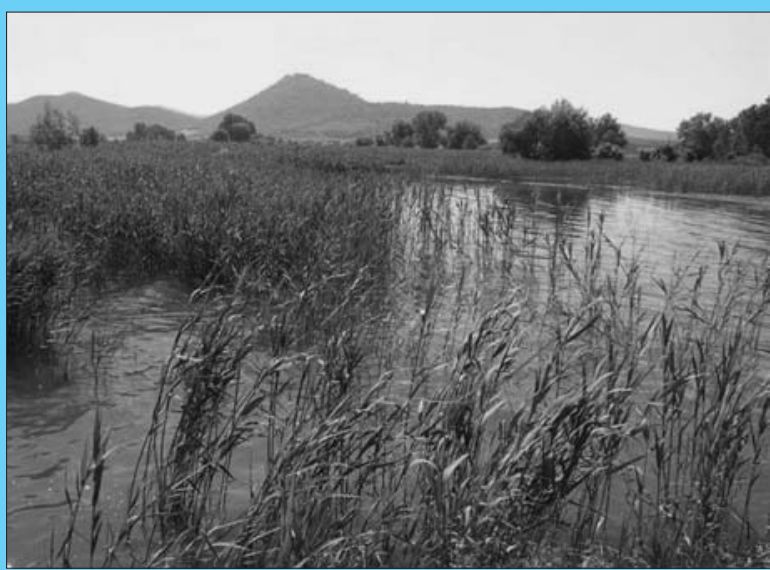

Figura 4. Particolare con canneti dell'attuale Lago Trasimeno

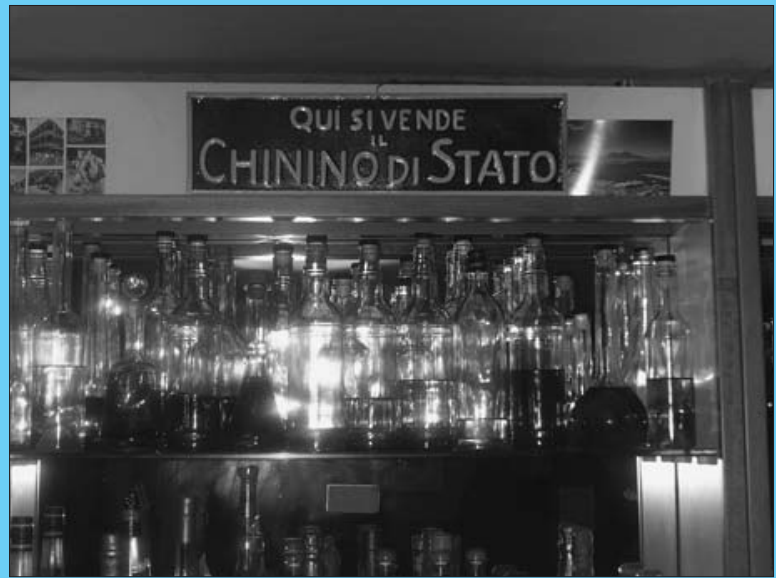

Figura 5. Vendita di Chinino di Stato in Privativa.

scena, con osservazioni e chiarimenti sul disseccamento del lago Trasimeno, quale quella di C. Bonfigli, e le 48 ragioni che condannavano invece il suo prosciugamento (presentate in un memoriale dalla Deputazione Provinciale dell'Umbria), il Consorzio venne formalmente costituito nel novembre 1877 , consorzio che si pose in netta contrapposizione con tutti coloro che volevano il prosciugamento del lago medesimo $(4,13$, 28).

Notevoli difficoltà sorsero subito e dovettero durare molti anni circa la legittimità giuridica dell'istituzione, soprattutto a seguito dei contrasti insorti con il Demanio dello Stato, proprietario del Lago, che aveva avanzato un ricorso su due articoli dello Statuto dell'Ente. Guido Pompili, presidente del Consiglio d'Amministrazione del Consorzio si adoperò per superare tali difficoltà. Ma ecco di seguito tralci delle sue fondamentali e memorabili "osservazioni e schiarimenti intorno al Consorzio costituito" del maggio 1879: “... II lago Trasimeno, per mancanza d'un emissario capace di mantenerlo ad un limite costante, va soggetto a variazioni... . Esso porta seco ... un'altra terribile calamità, la febbre palustre. Le acque sottilmente distese sopra le pianura allagate, ai primi raggi veramente cocenti del sole di giugno, si ritraggono, lasciando ivi a putrefarsi le erbe e tutte quelle materie, dalle esalazioni delle quali l'aria viene ammorbata ed infetta; donde poi hanno origine quelle pestilenze e quelle febbri per le quali il territorio d'una popolazione robusta e floridissima si muta in un ospedale di gente grama, sfiorita, malaticcia, che paga cosi dolorosamente una colpa non sua. E di chi è la colpa? ... la colpa è senza dubbio e per unanime consenso del proprietario del lago, il quale avrebbe dovuto provvedere... II proprietario del lago è il Demanio dello Stato... In seguito al voto unanime ... Consorzio... venne con decreto omologato dalla Potestà governativa definitivamente costituito e creato ente morale. ... II fatto è questo. II Consiglio superiore dei lavori pubblici, ..., non curò affatto il Consorzio ed il suo statuto ..., ma si occupò invece d'altra questione ad essi estranea, già tante volte agitata e che reputatasi ormai sepolta per sempre; vogliam dire del prosciugamento del lago. A questo esso si palesò inchinevole ... II disseccamento del lago,... errore, il pericolo ed il danno. Un lago è uno dei più sublimi e sorridenti spettacoli della natura, e l'uomo che non vive di solo pane e non è nato semplice mercante, si sente rapito alla sua vista... I laghi sono stati sempre fonti di poesia...

Da una poesia diV.Aganoor, da cui il lago è stato amato per le "visioni di bellezza e i sogni d'amore", ma, più ancora, perché testimone della tenace lotta per il suo bene da parte del proprio compagno di vita G. Pompili:

Dove anzi impregnavan le vie

Sue brezze i palustri veleni, la zappa giocondi baleni invia dalle uberrime vie;

\section{E il Pompili prosegue:}

Le altre nazioni, in generale più abbondanti di laghi che non sia l'Italia, non paiono prese da questa mania che abbiamo noi di prosciugarli; eppure non possiamo dire che non sappiano fare meglio di noi i loro interessi. Ma forse là non si sente da tanti il bisogno d'arricchirsi subitaneamente colle grandi concessioni e coi giganteschi lavori; perché le industrie ordinarie e la voglia di lavorar seriamente conducono più presto gli uomini ad abbeverarsi alle fonti salutari della vera e non della falsa e fallace ricchezza. ...Ma se a priori ... non deve mai, secondo noi, asciugarsi un lago, possono bensi darsi certe condizioni e certe ragioni che rendano necessario od immensamente ed incontrovertibilmente utile il suo asciugamento. Queste sono, quando il lago, per il suo stato idraulico, arreca gravi danni alla salute ed economia pubblica, ovvero quando col disseccamento possono ritrarsi vantaggi certi e reali grandissimi e di gran lunga superiori a quelli derivanti dalla sua esistenza. Noi ammettiamo ciò francamente, perché, mentre non siamo così bestialmente servi degli appetiti materiali da pesare ogni cosa sulla bilancia del dare e dell'avere, e ridurre tutto, anche la storia e la civiltà d'un popolo, ad una partita di ragioniere; ... Ch'esso sia esiziale, nelle sue condizioni presenti, alla pubblica salute ed ai patrimonii circostanti, è cosa indubitabile e ne fanno dolorosa prova quei medesimi pe' quali adesso scriviamo, tra cui già serpeggiano i brividi delle nefaste febbri esalanti dai loro terreni miseramente sommersi e devastati. Ma è ugualmente indubitabile che il Consorzio da essi costituito e di essi composto, se altri ostacoli non gli si pareranno innanzi, è già alla vigilia di cominciar l'opera che in brevissimo spazio di tempo toglierà le alluvioni e fermerà le acque durabilmente ad un limite costante, facendo cessare per sempre ogni danno ed 
ogni male, e lasciando intatto il lago, il quale quindi può essere bonificato senza essere asciugato; anzi più sotto mostreremo che, asciugandolo, per avventura non si bonificherebbe. Dunque la necessità del prosciugamento non esiste; ... (col prosciugamento) gli olivi stupendi e rigogliosissimi, ..., andrebbero tutti a male, ... Dallo scoprimento del fondo del lago ben lieve profitto trarrebbe il mercato del grano, del quale d'altra parte non c'è stata mai carestia; ma se n'avrebbe di certo la penuria del pesce,... A questi danni certi e incontrovertibili del prosciugamento, si aggiungono i pericoli d'altri maggiori ed irreparabili danni. ... II pensiero rifugge dai mali che, avverandosi il prognostico, nascerebbero da un prosciugamento avviato e non compiuto. L'Italia avrebbe perduto un bel lago testimonio di tante famose vicende, un buon capitale, stupendi olivi, squisito pesce, antiche arti, prospere industrie, e perché? Non già per acquistare agli uomini una pianura da coltivare, ma piuttosto alle ranocchie paludi e pantani da sguazzare, gracidando lor inni intorno al trono divenuto sacro e perpetuo della Dea Febbre. ... (II) Consorzio,..., ad ogni modo ha un'incontrastabile legale e legittima esistenza. Che cosa si propone...? Esso vuol togliere le presenti piene, riscattare i terreni sommersi e devastati dall'alluvione, ricondurre il lago al suo limite naturale e quivi mantenerlo costantemente, acciò che più non si rinnovellino i danni. Questo e non altro è il suo fine. ..." (25).

Dopo aspre ed estenuanti battaglie solo nel 1888 si costitui legalmente il "Consorzio degli interessati per la bonifica delle sponde del lago Trasimeno". Nel 1894 venne approvato un nuovo statuto e l'ente si chiamò "Consorzio speciale per la bonifica delle gronde del lago Trasimeno in provincia di Perugia". Lo scopo era quello di provvedere alla sistemazione del lago Trasimeno, in modo da impedire le espansioni permanenti sulle gronde e quindi di ottenere il risanamento igienico e la bonifica agricola del territorio interessato con il concorso dello Stato, della Provincia, dei comuni (28). Iniziarono così i lavori, con tanto di progetto, approvazione e così via. II 3 I dicembre 1899 viene pubblicata una lunga e minuziosa relazione generale sullo stato dei lavori per la costruzione del nuovo emissario, a firma dell'Ing. Menchini, Direttore dei Lavori (20).

Nel 1910, in occasione della commemorazione di Guido Pompili, il sindaco di Castiglione del Lago si espresse così: “... correvano anni tristi per questa regione (prima dell'istituzione del Consorzio), lo squallore delle campagne limitrofe al lago, sistematicamente e largamente inondate e la febbre, dea indisturbata tra le popolazioni rivierasche che ne avvizziva prematuramente il fiore della giovinezza e ne spezzava la fibra ancora salda e virile .... Fu allora che un giovane di 21 anni inalberò il vessillo redentore della iniziativa privata .... Oggi la malaria e la febbre sono per sempre bandite da questi declivi ubertosi, da questa chiostra di colli...." (38).

Questa una piccola parte della storia del "Lago salvato" (3I). Con la legge della regione Umbria n. 6 del 23 gennaio 1980, il Consorzio di bonifica del Trasimeno fu soppresso e le sue funzioni furono delegate alla Provincia di Perugia (Figura VI).

La malaria nell'area di Terni: da Piediluco alla Conca Ternana attraverso la Valnerina

La fondazione di Terni si fa risalire a svariati secoli prima di Cristo. I primi insediamenti sono probabil-

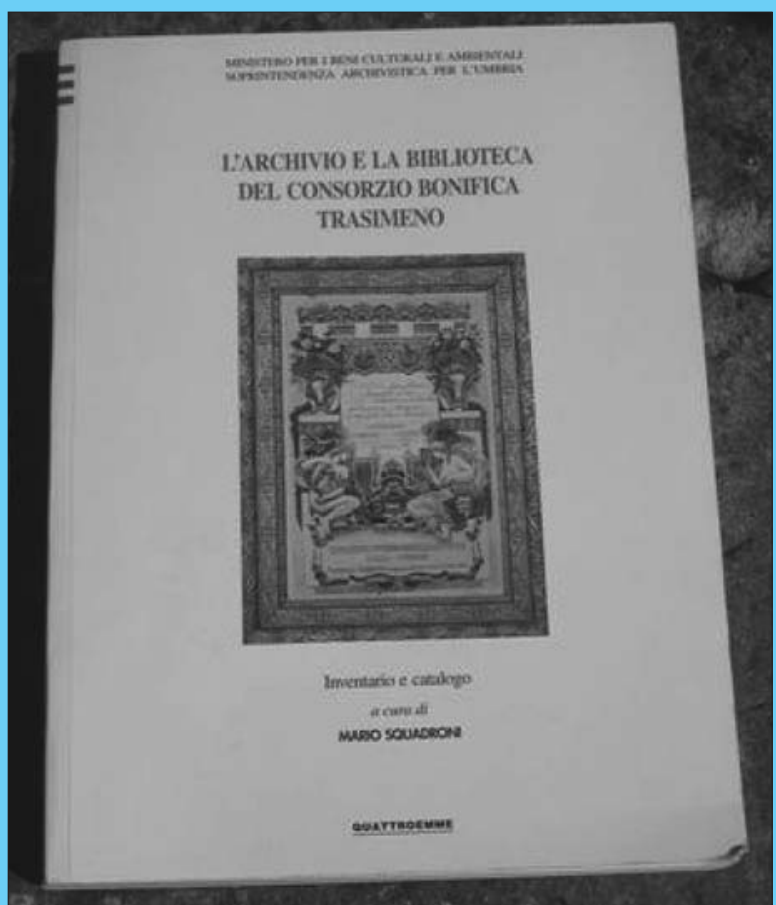

Figura 6. Il Catalogo dell'Archivio Storico del Consorzio di Bonifica del Trasimeno

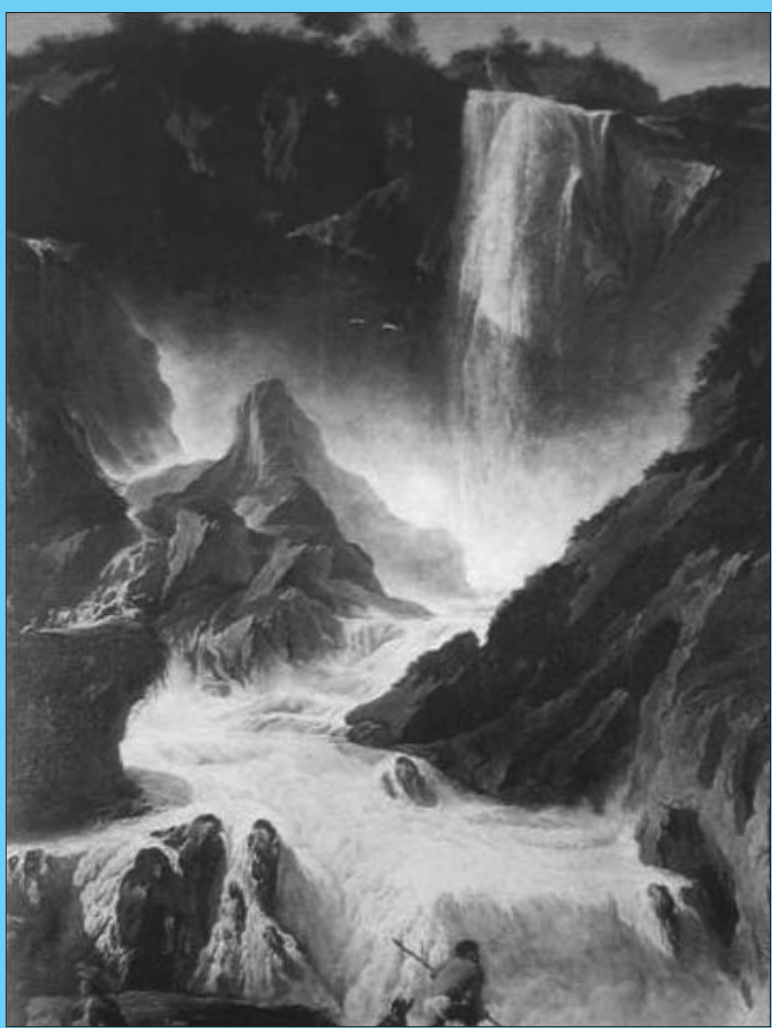

Figura 7. Le Cascate delle Marmore in un Olio su tela d’epoca (attribuito a P. P. Roos)

mente ascrivibili agli Umbri e/o ai Sabini, attorno al VIII - VII secolo A. C. E' sulla collina di Pentima che ebbe origine il primo insediamento umano nella conca ternana, dal momento che quest'ultima era allora in gran parte paludosa e occupata da un lago formato dal 


\section{RASSEGNA}

fiume Nera (I2). Intorno al IV - III secolo A. C. arrivano i Romani. E proprio ai Romani si deve il primo nome che ebbe la città: "Interamna", ovvero città posta tra due fiumi, il Velino e il Serra, quest'ultimo a carattere torrentizio e verosimilmente deviato in epoca medioevale (I).

La storia di questi due fiumi affonda nella notte dei tempi. Con una certa sicurezza si può parlare dell'esistenza, nell'era antropozoica, di un Lago Velino ("Lacus Velinus") formato con le acque del fiume omonimo, che aveva sommerso tutti i laghi dell'altopiano umbro-sabino. Le acque del Velino, molto ricche di sostanze calcaree, tracimando lungo tutta la larghezza del ciglione dell'altopiano, con i propri depositi continuavano a rialzare lo sbarramento delle "marmore", sommergendo nuove terre asciutte sino a giungere alla quota di circa 400 metri s. I. m. In seguito a grandiosi nuovi eventi naturali, il territorio si modificò ulteriormente, le acque del lago preistorico si aprirono un varco verso quelle del fiume Nera, il lago si svuotò parzialmente, e il livello scese si alcuni metri. La palude però prevalse ancora su gran parte del territorio (26).

Nel III - II secolo A. C. avviene la prima bonifica ad opera del Console romano Manlio Curio Dentato. Le acque calcaree del Velino, come detto, depositandosi costituivano una barriera per la confluenza con il fiume Nera, e si impaludavano sull'altopiano delle Marmore (un'ipotesi dell'etimologia del nome deriva dalla pietra calcarea, appunto, che costituisce l'altopiano) (I2). Questo fiume, "la/il Nera/Narco", nasce dall'alto Appennino centrale umbro-marchigiano, da "due fori, quasi orificii del naso del bove, ..., traendo" ... da ciò il nome di "Nahars o Nars", poi "Nare", Narco, ed infine Nera (I). II fiume Velino, "così detto dalla Dea Velia", trae origine nell'Appennino sabino-abruzzese, e, superata, Rieti, attraversa una piana sino a Piediluco, sopra la città di Terni, ove "... ibi erant palustria, quae nunc prisco linguae more dicuntur Velia" ("erano ivi degli acquitrini o pantani, che ora all'antico uso della lingua si dicono Velia"; da D. Alicarnasso, lib. I) (I).

Come in precedenza riferito, l'impaludamento delle piane sabine ed umbro-sabine era causa periodica di febbri malariche. M. C. Dentato bonificò i terreni circostanti il Velino, causa di tali straripamenti, scavando un canale che partiva dal punto più profondo della palude e che portava le acque fino al ciglione di Marmore. II canale ottenne l'obiettivo prefissato, ma risultò col tempo insufficiente per contenere le acque del Velino stesso nei periodi di piena, con conseguenti allagamenti a valle, lungo il decorso del Nera che dopo non molti chilometri passava per la città di Terni. Successivamente i reatini proposero comunque un ampliamento del canale per evitare lo straripamento delVelino, opera cui si opposero i ternani, che temevano inondazioni del loro territorio. Ciò diede luogo, per tutta l'età classica, a lunghi contenziosi tra i due municipi.

Ad ogni buon conto, con l'esecuzione iniziale del "Cavo Curiano", "la mano dell'uomo aveva profondamente mutato la natura, aveva inalveato le acque stagnanti, pestilenti e malariche, aveva effettuato la prima grande operazione di ecologia che la storia ricordi" (26).

Tra il XIV e il XV secolo D. C., l'innalzamento del fondo del canale scavato da Curio Dentato diminuì a tal punto lo scolo delle acque da rendere drammatica la situazione e riaprire lo scontro tra reatini e ternani.
Nel I4I7 iniziò l'escavazione di un nuovo canale, detto "Reatino", che, malgrado i successivi rimannegiamenti operati sotto il pontificato di Gregorio XIII nel XVI secolo, non funzionò mai bene. Nel dicembre 1545 Paolo III incaricò Antonio Sangallo il Giovane di costruire un nuovo canale, ultimato nel 1546 e denominato "Paolino", anche se neppur questo riuscì nello scopo. I documenti dicono che la costruzione di questo terzo cavo fu iniziato l'I I dicembre I545 alla presenza del Sangallo stesso che aveva preso dimora in una osteria di Piediluco. Ma il 28 settembre 1546 il Sangallo muore; l'architetto diresse così soltanto per una decina di mesi i lavori a Marmore, e pare proprio che la causa della sua morte sia stata causata dai miasmi malarici esalati dagli "acquitrini marmorei" poiché si dà per certo che l'impaludamento aveva portato come conseguenza anche la malaria (26).

Nel 1596 un altro papa, Clemente VIII, affidò a G. Fontana la riattivazione del canale d'età romana. Questi rese il canale più profondo, ne aumentò la pendenza, ne rettificò il percorso e costruì un Ponte Regolatore che avrebbe dovuto consentire il passaggio solo d'una determinata quantità di acqua. I lavori vennero ultimati nel I60I e il nuovo canale venne denominato "Clementino". Nonostante il funzionamento del Ponte Regolatore fosse tutt'altro che efficace, il nuovo canale risolse il problema principale: l'impaludamento della piana reatina. Restava invece irrisolto una altro problema: quando il Velino in piena precipitava nel Nera, l'acqua tracimava per oltre 7 chilometri nella Valnerina. Occorreranno molteplici studi e numerosi interventi prima che l'architetto Andrea Vici nel I7871788 trovasse la soluzione: un taglio diagonale sul secondo balzo che deviava parte dell'acqua in caduta, consentendo un migliore deflusso del Nera. Questo intervento diede alla Cascata (delle Marmore) il suo aspetto definitivo e l'attuale (35, 36; Figura VII).

II Velino si getta nel Nera dalla località di Marmore a quella sottostante di Collestatte con un salto di oltre 160 metri ("trecento piedi"). Siamo in Valnerina. Risalendola, in pochi chilometri si arriva a Ferentillo; da qui la valle si restringe ed entra in territorio perugino. Scendendo invece a valle, dopo sette chilometri, si arriva a Terni e ci si immette nella Conca Ternana.

\section{La cascata delle Marmore}

Da secoli gli abitanti della zona amano raccontare ai propri bambini una leggenda che si perde nella notte dei tempi. Questa narra che la bella ninfa Nera, innamorata del giovane pastore Velino, fu punita dalla Dea Giunone per la sua insolenza e trasformata in fiume, il Nera appunto. II bel Velino, disperato per la perdita dell'amata, si gettò dalla rupe di Marmore per ricongiungersi ad essa: il salto mortale sarebbe continuato per l'eternità e avrebbe dato vita alla meravigliosa Cascata delle Marmore.

Nel cuore della Valnerina perugina (la Valnerina si forma presso Visso, nella marca maceratese), ecco cosa recita un "depliant" turistico, a proposito degli itinerari benedettini ivi presenti numerosi e della loro storia: "scendendo a valle e lasciati alle spalle i borghi fortificati di Cerreto e Vallo di Nera, s'incontra il piccolo centro di Castel S. Felice, arroccato su una piccola altura. Alle sue pendici l'Abbazia di S. Felice si staglia con la bella facciata romanica contro il verde smagliante dei boschi. Sotto la maestosa abside, alleggerita da 


\section{RASSEGNA}

lesene e archetti pensili, scorre tranquillo il Nera, svogliato come un gigante assopito per "farsi perdonare" le disastrose piene che, prima dell'arrivo dei benedettini, inondavano la valle circostante, distruggendo il raccolto dei contadini e creando vaste zone paludose dove dilagava la malaria".

Ricordo ancora come sullo stemma della città di Terni è raffigurato un drago, detto Tiro (Thyrus). Questa simbologia è legata ad una leggenda che narra di un orribile drago che, tanti anni addietro, viveva nel territorio ternano, tenendo in continuo terrore tutta la popolazione. Finalmente un giorno un giovane ternano di una nobile famiglia (i Cittadini) riuscì a sconfiggere il drago, ottenendo in dono i terreni che un tempo erano stati infestati dal drago stesso, luoghi che in breve divennero tra i più ameni del territorio. Probabilmente questa leggenda ebbe origine dal fatto che, un tempo, gran parte di tale territorio era paludoso. Poi i terreni vennero prosciugati dalla bonifica, divennero fertili e belli. Così, gli acquitrini e la malaria rimasero solo un lugubre ricordo e si identificarono, nella fantasia popolare, con la figura del drago.

Ma leggende non son di rado frutto di verità storiche. La malaria è sicuramente stata presente anche nella Conca Ternana vera e propria. Se difficile è reperire documenti comprovanti con certezza tale stato di cose (seppure, come in precedenza riportato, alcuni numeri e referti esistono), alcune osservazioni recenti scientifiche potrebbero avvalorare tutto ciò. Intendo dire la presenza del gene beta-talassemico nella popolazione del ternano.

In una breve serie successiva di studi, alcuni Autori negli anni '80 del $\mathrm{XX}$ secolo hanno individuato tra l'। e il $2 \%$ della popolazione esaminata la presenza del "trait" eterozigote per la beta-talassemia $(5,16)$. La provincia di Terni e, soprattutto, la zona compresa tra Terni e Rieti possiedono le caratteristiche che potrebbero aver permesso il mantenimento e lo sviluppo della tara talassemica. Tale regione, in passato malarica, comprende infatti l'antico territorio, come detto, del Lacus Velinus, che fino al tardo Medio Evo con stagni ed acquitrini si estendeva dal piano di Marmore a Rieti. II ritrovamento di svariati casi portatori del "trait" talassemico non sembra così essere conseguenza dei flussi migratori da regioni ad alta incidenza (16). La persistenza del gene talassemico, infatti, è giustificata dalle caratteristiche di tale territorio, un tempo paludoso, in quanto la presenza di siffatto gene nella forma eterozigote rappresenta un privilegio nelle aree ad endemia malarica (I7). Pertanto l'esistenza del "trait" betatalassemico è di origine essenzialmente autoctona, sebbene alcuni flussi migratori da altre regioni vi siano stati, soprattutto nel capoluogo della provincia, ove un incremento demografico importante si realizzò a cavallo tra il XIX e XX secolo in ragione dello sviluppo industriale che in quei decenni vi fu (I7). Ciononostante, in linea di massima, continuano gli studiosi, tale territorio, fuori città in particolar modo, non è mai stato punto di riferimento per lo spostamento di masse, fatta eccezion, come accennato, ma solo in parte, per la città di Terni. E la crescita demografica del Comune di Terni è poi solo in parte in relazione all'immigrazione di altre province, essendo questa legata principalmente all'inurbamento delle popolazioni rurali e montane circostanti (I6).
Pertanto la presenza del "trait" talassemico è verosimilmente in relazione a mutazioni avvenute in tale circoscritto territorio (16).

Per concludere, nonostante le varie opere realizzate nel corso dei secoli, a partire dal "Cavo Curiano" a quello "Paolino", la zona era rimasta malarica e gli scavi medesimi avevano reso insalubri anche le piane sottostanti della Valnerina (I7). Le caratteristiche del territorio, di fatto collocato al centro di cinque vallate, in passato paludose e malariche, possono giustificare la presenza di portatori autoctoni del "trait", se si ipotizza la beta-talassemia come originata attraverso mutazioni non avvenute in un singolo focus, ma in varie località (I7).

\section{Considerazioni conclusive}

Il presente scritto non vuole avere la pretesa di essere esaustivo né rispecchiare con certezza la realtà delle cose. Ho riportato quanto sono riuscito a reperire in alcune biblioteche (di Perugia e di Castiglione del Lago) e negli Archivi Storici di alcuni Comuni dell'Umbria.

Sicuramente anche in altre zone la malaria è stata in qualche modo presente: nell'Orvietano (zona anch'essa a suo tempo ricca di canapaie, per esempio), nella vallata ove il fiume Paglia si unisce al Chiani; e più a sud, oltre l'attuale Lago di Corbara, a monte ed a valle dell'odierna Oasi di Alviano (sempre lungo il decorso del Tevere), nei pressi di Lugnano in Teverina ove già in una precedente nota riportavo l'ipotesi di pregresse (e gravi) epidemie malariche $(3$, II). In ogni caso il problema della malaria, in questa Regione, è stato sicuramente molto meno importante di altre calamità naturali o altre malattie (infettive/contagiose e non) di ben più vasta portata, vuoi nella loro diffusione vuoi nella loro gravità, nel corso dei secoli, e basti pensare al tifo, al colera, al vaiolo, alla pellagra, e via discorrendo.

In ogni caso spero di aver per sommi capi dato un'immagine interessante, o comunque suggestiva, di quanto possa essere accaduto a causa, per e in conseguenza di tale patologia parassitaria (e come tale inquadrata soltanto in tempi più recenti), di indubbia importanza nella storia medica e sociale italiana dei secoli passati.

\section{Ringraziamenti}

Un prezioso e determinante aiuto mi è stato dato da varie persone, alcune delle quali conosciute nel corso di tale breve ricerca. Un sentito grazie, soprattutto, a Elena Teatini e Paolo Magionami di Castiglione del Lago e a Maria Chiara Medori di Terni, che mi hanno fornito materiale fondamentale per potere scrivere tutto ciò. Ma un grazie anche ad altre persone, non foss'altro perché sono state comunque disponibili, laddove non sempre altre hanno sentito il bisogno di farlo; un grazie allora a Maria Letizia D'Annibale (Terni), Anna Napoleoni (S.Anatolia di Narco), Silvia Faloci (Perugia), Paolo Bianchi (Spoleto) e Sabrina Caciotto (Panicale).

\section{BIBLIOGRAFIA}

I. Angeloni F. Storia di terni. Edizioni Thyrus, Terni, III Edizione, 1987.

2. Binacchiella E. Castiglione del Lago e il suo territorio. Tipo-Lito Missioni Estere, Perugia, 1972. 
3. Bonelli F. Evoluzione demografica ed ambiente economico nelle Marche e nell'Umbria dell'Ottocento. ILTE, Torino, 1967.

4. Bonfigli C. Osservazioni e schiarimenti sul disseccamento del Lago Trasimeno. Unione Tipografico-Editrice, Torino, 1864.

5. Brancali P. L., Materazzi M., Medori M. C., Prete C., Lorenzoni L., Campili E.Valutazione della frequenza all'elica del gene della beta-talassemia in un campione della popolazione della Conca Terzana. Nuovi Annali di Igiene e Microbiologia, Società editrice Universo, Roma, 1986.

6. Brandel F. Civiltà e imperi del Mediterraneo nell'età di Filippo II. Einaudi, Torino, 1953.

7. Casagrandi O., Guzzon L. Nozioni di igiene e di legislazione per la lotta antimalarica. (Scuola di Scienze Politiche e Sociali della R. Università di Padova. Corso di preparazione per funzionari amministrativi dei Consorzi di bonifica), III Edizione, Cedam, Padova, 1929.

8. Celli A. La malaria secondo le nuove ricerche. Edizioni Alighieri, Roma, 1899.

9. Coppioli L. Guida sanitaria dell'Umbria. L’Umbria idrologica e climatologia. Firenze, 1913.

10. Cosmacini G. Storia della medicina e della sanità in Italia. Economica Laterza, Bari, II Edizione, 1998.

II. Crotti D. La malaria, ossia la mal'aria: brevi note di una "storia sociale e popolare". Le Infezioni in Medicina, 2005.

12. Della Croce G. F., Massarelli R. Un viaggio in Umbria. Edimond, Città di Castello (PG), 2005.

13. Deputazione Provinciale dell'Umbria. Sul bonificamento del Lago Trasimeno ed il profitto delle sue acque. Stab. Tip. A. Nucci, Siena, 1867.

14. De Santis G., Lussana Grasselli E. L'infestazione malarica tra gli squilibri ambientali e insediamenti umani. In: Seminario Internazionale di Geografia Medica, I, Roma, 1982.

15. Desplanques H. Campagne umbre; contributo allo studio dei paesaggi rurali dell'Italia Centrale. Quaderni Regione dell'Umbria, 1971.

16. Fatati G., Lorenzoni L., Materazzi M., Medori M.C., Palazzesi G.P. Screening per la talassemia nella provincia di Terni. La Trasfusione di Sangue, Vol. XXXIII - N. 5, II Pensiero Scientifico Editore, Roma, 1988.

17. Fatati G., Palazzesi G.P., Cassetti M., Lorenzoni L., Coaccioli S., Puxeddu A. Beta-talassemia: motivazione per uno Screening a Terni. Minerva Medica 75, Torino, 1884.

18. Giampaoli G. La canapa. EFFE, Perugia, 2004.

19. Grassi G.B. La malaria propagata da particolari zanzare. Trever, Milano, 1900.

20. Innamorati L. Poche osservazioni sulla relazione della Commissione eletta dal Consiglio Provinciale dell'Umbria per riferire sulla convenienza del prosciugamento del Lago Trasimeno. Tipografia Cantucci, Perugia, 1864.

2I. Majori G., Bettini S. I'Istituto Superiore di Sanità e l'eradicazione della malaria in Italia. G. It. Med. Trop., 6, 200 I.

22. Mariotti A. Riflessioni fisico-mediche sul progetto del disseccamento del lago Trasimeno. Perugia, 3 ottobre 1789.

23. Menchini L. Consorzio del Trasimeno. Costituzione del nuovo emissario. Relazione generale della Direzione dei Lavori al Comitato Esecutivo del Consorzio. Unione Tipografica Cooperativa, Perugia, 1900.

24. Natali F. Un'escursione intorno al Trasimeno. Tipografia Tomassini, Foligno, 1874.

25. Pompili G. Osservazioni e schiarimenti intorno al Consorzio costituto per sistemare il Lago Trasimeno.
Tipografia V. Bartelli, Perugia, 1879.

26. Secci T. Disegni e stanze della Cascata delle Marmore dal I545 al 1976. Umbriagraf, Terni, 1980.

27. Serpieri A. La bonifica nella storia e nella dottrina. Ed agricole, Bologna, II Edizione, 197I.

28. Squadroni M. (a cura di). L'archivio e la biblioteca del Consorzio Bonifica del Trasimeno. Ministero per i beni Culturali e ambientali; Sopraintendenza Archivistica per I'Umbria. Quattroemme, Perugia, 1996.

29. Taricone F. (a cura di). II lavoro femminile nell'Ottocento e nel Novecento: le tabacchine. Gangemi Editore, Roma 2005.

30. Teatini E.Ambiente, salute e calamità naturali nel territorio trasimenico nella prima metà dell'Ottocento. Università degli Studi di Perugia. Annali della facoltà di lettere e Filosofia. 2. Studi Storico-Antropoligici Volume XXXI-XXXII, nuova serie Volume XVII-XVIII, I 993-94 $1994 / 95$.

3I. Toccaceli E.E.Trasimeno. II lago salvato. Sellerio, palermo, 1990.

32. Torelli L. Carta della malaria dell'Italia. Pellai, Firenze, 1982.

33. Toscanelli N. La malaria nell'antichità e la fine degli Etruschi. Hoepli, Milano, 1927.

34. Trottarelli G. Memoria di Terni Igienica. Tipo-Litografia Cooperativa, Terni, 1899.

35. Umbria. Itinerari del Ternano. La Cascata delle Marmore. Umbriagraf, Terni, 2006.

36. Umbria. Itinerari del Ternano. Piediluco e il suo lago. Umbriagraf, Terni, 2006.

37. Unione Regionale Umbria. Bonifica integrale e montana in Umbria. Editrice San marco, Perugia, 1975.

38. Università degli Studi di Perugia. Facoltà di Lettere e Filosofia. Corso di laurea in lettere Moderne. Tesi di Laurea in Geografia: Ambiente, salute e calamità naturali nel territorio trasimenico nella prima metà dell'Ottocento. Laureanda E. Teatini, Relatore A. Melelli. Anno Accademico 1994-1995.

\section{Daniele Crotti}

Libero Professionista in Parassitologia

e Microbiologia Medica

Strada Comunale per Pilonico Paterno 4 - 06080

Pianello, Perugia

Tel.: 075602372

E-mail: nenedc@tin.it 